16. Klein, R. B., Fischer, T. J., Gard, S. E., Biberstein, M., and Steim, E. R. Decreased mononuclear and polymorphonuclear chemotaxis in newborns. Pediatrics, 60: 467 (1977).

17. Lancefield, R. C.: A micro-precipitin technique for classifying hemolytic streptococci and improved methods for producing antisera. Proc. Soc. Exp. Biol. Med., 38: 473 (1938)

18. Lash, J. A., Coats, T. D., Baehner, R. L., and Boxer, L. A.: Plasma lactoferrin reflects granulocyte activation in vivo. Pediatr. Res. (abstract), 16: 208A (1982)

19. Laurenti, F., LaGreca, G., Ferro, R., and Bucci, G.: Transfusion of polymorphonuclear neutrophils in a premature infant with Klebsiella sepsis. Lancet, 2: 111 (1978).

20. Laurenti, F., Ferro, R., Isacchi, G., Panero, A., Salvignono, P. G., Malagino, R., Palmero, D. and Bucci, G.: Polymorphonuclear leukocyte transfusion for the treatment of sepsis in the newborn infant. J. Pediatr., 98: 188 (1981).

21. McCracken G. H., Jr. and Eichenwald, H. F.: Leukocyte function and the development of opsonic and complement activity in the neonate. Am. J. Dis. Child., 121: 120 (1971).

22. Miller, M. E.: Host Defences in the Human Neonate. pp 1-3. Grune and Stratton Inc., New York, N.Y. (1978).

23. Miller, M. E.: Chemotactic function in the human neonate: Humoral and cellular aspects. Pediatr. Res., 5: 487 (1971).

24. Miller, M. E.: Pathology of chemotaxis and random mobility. Semin. Hematol., 12: 59 (1975).

25. Pincus, S. H., Ewing, L. P. and Stocks, C. J.: Anti -(T,G)-A--L Hybrdomas. Fine specificity and idiotypic characterization. Molecular Immunol., 19: 1551 (1982)

26. Rote, N. S., Taylor, N. L., Shigeoka, A. O., Scott, J. R., and Hill, H. R.: Enzymelinked immunosorbent assay for group B streptococcal antibodies. Infect. Immun., 27: 118 (1980).

27. Santos, J. I., Shigeoka, A. O., and Hill H. R.: Functional leukocyte administration in protection against experimental neonatal infection. Pediar. Res., 14: 1400 (1980).

28. Santos, J. I., Shigeoka, A. O., Rote, N. S., and Hill, H. R.: Protective efficacy of a modified immune serum globulin in experimental group B streptococcal infection. J. Pediatr., 99: 873 (1981)

29. Shigeoka, A. O., Pincus, S. H., Rote, N. S., and Hill H. R.: Hybridoma typespecific IgM antibody offers enhanced protection against systemic or respiratory group B streptococcal experimental infection. Pediatr. Res. (abstract), 16: 231A (1982).

30. Shigeoka, A. O., Hall, R. T., and Hill H. R.: Blood transfusion in group B streptococcal sepsis. Lancet, 1: 636 (1978).

31. Tollner, U., Pohlandt, F., Heinze, F., and Henrichs, I.: Treatment of septicemia in the newborn infant: choice of initial antimicrobial drugs and the role of exchange transfusion. Acta Paedictr. Scand., 66: 605 (1977).

32. Vain, N. E., Mazulmain, J. R., Swarner, O. W., and Cha, C. C.: Role of exchange transfusion in the treatment of severe septicemia. Pediatrics, 66: 693 (1980).

33. Wasserman, R. L.: Neonatal sepsis: the potential of granulocyte transfusion. Hosp. Pract., 17: 95 (1982).

34. Weissmann, G., Smolen, J. E., and Korchak, H. M.: Release of inflammatory mediators from stimulated neutrophils. N. Engl. J. Med., 303: 27 (1980).

35. The authors thank Nancy Taylor, Jayne Blair, Peter Viavant for technical assistance.

36. Requests for reprints should be addressed to: Robert D. Christensen, M.D Division of Hematology, University of Utah School of Medicine, 50 North Medical Drive, Salt Lake City, Utah, 84132.

37. Supported by two grants from the Thrasher Research Fund and by U.S. Public Health Service Grants \#HD-14419, AI-13150 and AI-19094

38. Received for publication October 6, 1982.

39. Accepted for publication February 18, 1983

\title{
Lymphocyte-Derived Chemotactic Factor Production by Neonatal Lymphocytes
}

\author{
MARGARET A. KELLER, ${ }^{(18)}$ ROSE M. KIDD, ROSEMARY D. LEAKE, AND SUSAN L. EVERETT \\ Department of Pediatrics, UCLA School of Medicine, Harbor-UCLA Medical Center, Torrance, California USA
}

\section{Summary}

We examined neonatal lymphocyte production of the lymphokine, Iymphocyte-derived chemotactic factor (LDCF). Supernatants from 10 neonatal lymphocyte cultures were paired with the supernatants from 10 adult lymphocyte cultures. Chemotactic activity was defined as the number of adult monocytes migrating toward phytohemagglutinin-stimulated supernatants minus the number of monocytes migrating toward nonstimulated control supernatants using a blind well chamber assay. Eight of the 10 neonatal lymphocyte cultures produced LDCF and five of the 10 adult lymphocyte cultures showed LDCF activity. The mean number of monocytes migrating toward neonatal supernatants was $13.0 \pm 1.5$ and toward adult supernatants was $14.1 \pm 2.1$. To determine if a quantitative difference in LDCF production did exist, six additional experiments were performed assaying multiple dilutions of supernatants. No evidence was found for a quantitative difference in neonatal LDCF production compared to adult production. Our studies show that neonatal mononuclear cells are functionally as competent as adult mononuclear cells to produce LDCF in response to a mitogen challenge.

\section{Abbreviations}

HBSS, Hanks' balance salt solution LDCF, lymphocyte-derived chemotactic factor
MIF, migration inhibition factor

PHA, phytohemagglutinin

PPD, tuberculin, purified protein derivative

The newborn infant's increased susceptibility to viral, fungal, and protozoal infections is consistent with impaired cellular immunity. Although absolute numbers of $\mathrm{T}$ cells and mitogen-induced $\mathrm{T}$ cell proliferation has been normal in peripheral blood obtained from neonates $(16,17)$, a number of investigators have documented impaired cutaneous hypersensitivity (16). Fireman et al. (8) have shown that neonates are deficient in their ability to show skin test reactivity to PPD antigen. The neonates studied were given transfer factor or leukocytes from PPD positive mothers. Their peripheral lymphocytes were then capable of responding to PPD by blastogenesis and capable of passively transfering skin test reactivity to adults yet these neonates could not develop a positive skin test themselves. These abnormal skin test responses may indicate a functional defect of neonatal lymphocytes or macrophages in producing important mediators of inflammation or may indicate impaired responses of neonatal monocytes and polymorphonuclear cells to inflammatory mediators.

Lymphokines, immunologic mediators produced by lymphocytes, have been shown to be an important component in delayed hypersensitivity (1), but it is still not clearly defined which lymphokine is responsible for the typical accumulation of mononu- 
clear cells in delayed type skin reactions. LDCF has been thought to be a potential mediator for this phenomenon $(5,13)$. Hence the study of LDCF production is particularly relevant to neonatal immunity.

Studies by previous investigators $(4,7,9-12,16)$ have yielded conflicting results concerning the functional capability of neonatal lymphocytes to produce lymphokines. In one study of MIF (16), the discrepancy between adult and neonatal lymphokine production was most evident when dilutions of lymphocyte culture supernatants were assayed, suggesting a quantitative difference in production between adult and neonatal specimens. In addition, discrepancies between cord lymphocyte and neonatal lymphocyte lymphokine production were reported (16). We examined neonatal lymphocyte production of the lymphokine, LDCF to further assess the functional capability of neonatal lymphocytes compared with adult lymphocyte controls and to further define the defect of cellular immunity in neonates.

\section{MATERIALS AND METHODS}

Heparinized peripheral blood $(10-15 \mathrm{U} / \mathrm{ml})$ was prepared from samples obtained by venipuncture from 10 neonates, 2-6 days of age, and from 10 healthy adult laboratory workers. Informed consent was obtained from the mother of each infant studied. In general, 3-5 cc of heparinized peripheral blood was obtained from these neonates.

Lymphocyte cultures. Lymphocyte cultures were prepared by Ficoll-Hypaque differential centrifugation of peripheral blood (3). Mononuclear cells were then washed three times with HBSS. A wet-preparation differential of mononuclear cells was done using toluidene blue stain, and $10^{6}$ lymphocyte $/ \mathrm{ml}$ were suspended in serum-free RPMI 1640 (25 mM Hepes buffer and L-glutamine, Gibco) with additional L-glutamine $(0.6 \mathrm{mg} / \mathrm{ml})$ plus an antibioticantimycotic preparation (Penicillin $100 \mathrm{unit} / \mathrm{ml}$, fungizone 0.25 $\mu \mathrm{g} / \mathrm{ml}$, streptomycin $(100 \mu \mathrm{g} / \mathrm{ml})$, and gentamicin $(10 \mu \mathrm{g} / \mathrm{ml})$. Lymphocytes were incubated with PHA (Burroughs Welcome) for $48 \mathrm{~h}$ because preliminary studies had determined $48 \mathrm{~h}$ as an optimal time to assay supernatants for LDCF. Total culture volume was $0.5-1.0 \mathrm{cc}$ at each PHA dose. The cultures were centrifuged at $400 \times g$ for $15 \mathrm{~min}$, and supernatants were aspirated and frozen at $-70^{\circ} \mathrm{C}$ for later assay. PHA was added to nonstimulated control cultures at the end of the 48-h incubation period, and these control supernatants were processed similarly.

Monocyte chemotaxis assay for LDCF. Lymphocyte culture supernatants were assayed for LDCF activity by the method of Altman et al. (2) and Snyderman et al (15). Blind well chambers (Bio-Rad Laboratories) were used with upper and lower compartments separated by a $5.0 \mu \mathrm{m}$ Nuclepore filter. Human peripheral monocytes from adult volunteers were prepared by Ficoll-Hypaque differential centrifugation. The wet-preparation differential was done using toluidene blue stain to ascertain monocyte content and the mononuclear cells were suspended at a concentration of $1.5 \times 10^{6}$ monocytes $/ \mathrm{ml}$ in the same serum-free media used for the lymphocyte cultures (RPMI-1640 with additional L-glutamine, antibiotic/antimycotic, and gentamicin). Appropriate dilutions of test or control supernatants $(0.2 \mathrm{ml})$ were placed in the lower compartments. All dilutions of supernatants used serum-free RPMI 1640. The monocyte suspensions $\left(0.2 \mathrm{ml}\right.$ of $1.5 \times 10^{6}$ monocytes $/ \mathrm{ml}$ ) were placed in the upper compartments. The chemotaxis chambers were incubated for $90 \mathrm{~min}$ at $37^{\circ} \mathrm{C}$ in $5 \%$ humidified $\mathrm{CO}_{2}$. The filters were removed, fixed with ethanol, and stained with hematoxylin and Wright stain. For each filter, the number of monocytes migrating through the filter toward test supernatants was counted under oil immersion in 20 fields. The mean number of migrating monocytes per oil field was determined. Filters were tested in triplicate.

For each assay, control solutions were also assayed for monocyte chemotactic activity to determine response of test monocytes. Controls were serum-free RPMI 1640 as described above and endotoxin (Salmonella typhosa, Sigma) activated pooled plasma diluted 1:10. All monocyte suspensions used responded to endo- toxin activated plasma when compared to serum-free RPMI 1640.

Chemotactic activity was defined as the number of monocytes migrating toward stimulated supernatants minus the number of monocytes migrating toward nonstimulated control supernatants. Student's $t$ test (6) was used to compare monocyte migration toward stimulated supernatants with migration toward control supernatants.

\section{RESULTS}

Cell composition of lymphocyte cultures. Neonatal and adult control mononuclear cell preparations were similar in cellular composition when a wet-preparation differential was done using toluidene blue stain. Neonatal mononuclear preparations contained $72.7 \pm 1.5 \%$ lymphocytes, $26.4 \pm 1.4 \%$ monocytes, and 0.8 $\pm 0.3 \%$ polymorphonuclear cells. Adult control preparations contained $77.5 \pm 1.3 \%$ lymphocytes, $21.5 \pm 1.1 \%$ monocytes, and 1.0 $\pm 0.3 \%$ polymorphonuclear cells.

Dose response. PHA dose response was determined with PHA concentrations from $0.03-3.3 \mu \mathrm{g} / \mathrm{ml}$. Maximal LDCF production for both neonatal and adult lymphocytes cultures occurred in the range of $0.03-0.8 \mu \mathrm{g} / \mathrm{ml}$. Although the PHA dose for maximal response varied, lymphocytes were incubated with at least two and usually three or four concentrations of PHA in the peak response range. Supernatants from each dose were assayed with the supernatant from the appropriate control culture.

Experiments with both neonatal and adult lymphocyte cultures determined $48 \mathrm{~h}$ as an optimal time to harvest supernatants. Prior experiments with adult lymphocyte cultures determined that a 1:3 dilution of supernatant was optimal for LDCF assay in this system.

$L D C F$ production. Supernatants from 10 neonatal lymphocyte cultures were paired with the supernatants from 10 adult lymphocyte cultures. Supernatants from each pair were assayed simultaneously for LDCF activity using the same adult test monocytes. A 1:3 dilution of supernatant was used. Results from these studies are summarized in Table 1. Eight of the 10 neonatal lymphocyte cultures produced LDCF and five of the 10 adult lymphocyte cultures showed LDCF activity. The mean number of monocytes migrating toward neonatal supernatants was $13.0 \pm 1.5$ and toward adult supernatants was $14.1 \pm 2.1$. Supernatants were considered positive for LDCF if the number of monocytes migrating toward stimulated supernatants was statistically different from the number of monocytes migrating toward control supernatants using Student's $t$ test $(P \leq 0.05)$. In some studies variation in triplicate filters did not allow results to be statistically significant although mean number (mean of three filters) of monocytes migrating toward stimulated supernatants did exceed mean number migrating toward control supernatants.

Although these studies used only a 1:3 dilution of lymphocyte supernatants, a quantitative difference in neonatal versus adult LDCF production might not have been observed. To determine if a quantitative difference in LDCF production did exist, six additional experiments were performed assaying undiluted supernatants and $1: 2,1: 3,1: 5$, and $1: 10$ dilutions of culture supernatants. Either a PHA concentration of 0.08 or $0.8 \mu \mathrm{g} / \mathrm{ml}$ was used, and again all experiments were done with adult-neonatal pairs. No evidence was found for a quantitative difference in LDCF production. Higher dilutions of neonatal supernatants did not lose chemotactic activity when compared to adult supernatant dilutions. Two sample dilution curves are shown in Figure 1. Each experiment represents one adult-neonatal pair.

\section{DISCUSSION}

Earlier published studies of cord and neonatal lymphokine production have yielded conflicting results about the functional capability of these mononuclear cells. Stiehm et al. (16) found both neonatal and cord mononuclear cells to be deficient in MIF production when stimulated with PHA, with neonatal lymphocytes being quantitatively more deficient than cord lymphocytes. 
Table 1. LDCF production by neonatal and adult lymphocyte cultures, No. of migrating monocytes \pm S.E.M./oil immersion field

\begin{tabular}{|c|c|c|c|c|c|c|}
\hline \multirow[b]{2}{*}{ Expt. No. } & \multicolumn{3}{|c|}{$\begin{array}{c}\text { Neonatal Supernatants } \\
\text { 1:3 dilution }\end{array}$} & \multicolumn{3}{|c|}{$\begin{array}{l}\text { Adult Supernatants } \\
\text { 1:3 dilution }\end{array}$} \\
\hline & $\mathrm{NS}^{1}$ & $\mathrm{~S}^{2}$ & $\Delta^{3}$ & NS & $\mathbf{S}$ & $\Delta$ \\
\hline 2 & $15.8 \pm 3.8$ & $29.1 \pm 1.5$ & $13.3 \pm 4.1^{4}$ & $18.3 \pm 3.5$ & $27.3 \pm 7.7$ & $9.0 \pm 8.4$ \\
\hline 3 & $5.5 \pm 2.2$ & $12.4 \pm 2.0$ & $6.9 \pm 3.0^{4}$ & $7.7 \pm 1.9$ & $14.2 \pm 1.7$ & $6.5 \pm 2.5^{4}$ \\
\hline 4 & $25.4 \pm 3.6$ & $42.0 \pm 1.9$ & $16.6 \pm 4.1^{4}$ & $12.8 \pm 6.5$ & $18.3 \pm 3.4$ & $5.5 \pm 7.3$ \\
\hline 7 & $7.8 \pm 3.4$ & $40.1 \pm 3.1$ & $32.3 \pm 4.6^{4}$ & $15.0 \pm 6.4$ & $52.8 \pm 6.8$ & $37.8 \pm 9.3^{4}$ \\
\hline 8 & $2.6 \pm 1.2$ & $12.8 \pm 5.1$ & $10.2 \pm 5.2$ & $1.7 \pm 0.7$ & $8.4 \pm 4.6$ & $6.7 \pm 4.7$ \\
\hline 9 & $4.4 \pm 1.7$ & $11.1 \pm 0.8$ & $6.7 \pm 1.9^{4}$ & $2.0 \pm 0.7$ & $14.1 \pm 5.6$ & $12.1 \pm 5.6^{4}$ \\
\hline 10 & $11.5 \pm 3.2$ & $22.3 \pm 3.0$ & $10.8 \pm 4.4^{4}$ & $8.3 \pm 3.1$ & $25.0 \pm 8.8$ & $16.7 \pm 9.3$ \\
\hline
\end{tabular}

${ }^{1}$ Mean number of monocytes/oil field migrating toward control supernatants [phytohemagglutinin (PHA) added to culture at end of 48-h incubation]. Mean is mean of triplicate filters.

${ }^{2}$ Mean number of monocytes/oil field migrating toward supernatants from PHA-stimulated cultures. Maximal responses are listed. PHA ranged from $0.03-0.8 \mu \mathrm{g} / \mathrm{ml}$.

${ }^{3} \Delta$ indicates the number of monocytes migrating toward stimulated supernatants minus number of monocytes migrating toward control supernatants.

${ }^{4}$ Indicates $P \leq 0.05$ by Student's $t$ test when the number of monocytes migrating toward stimulated supernatants was compared to the number migrating toward nonstimulated supernatants.
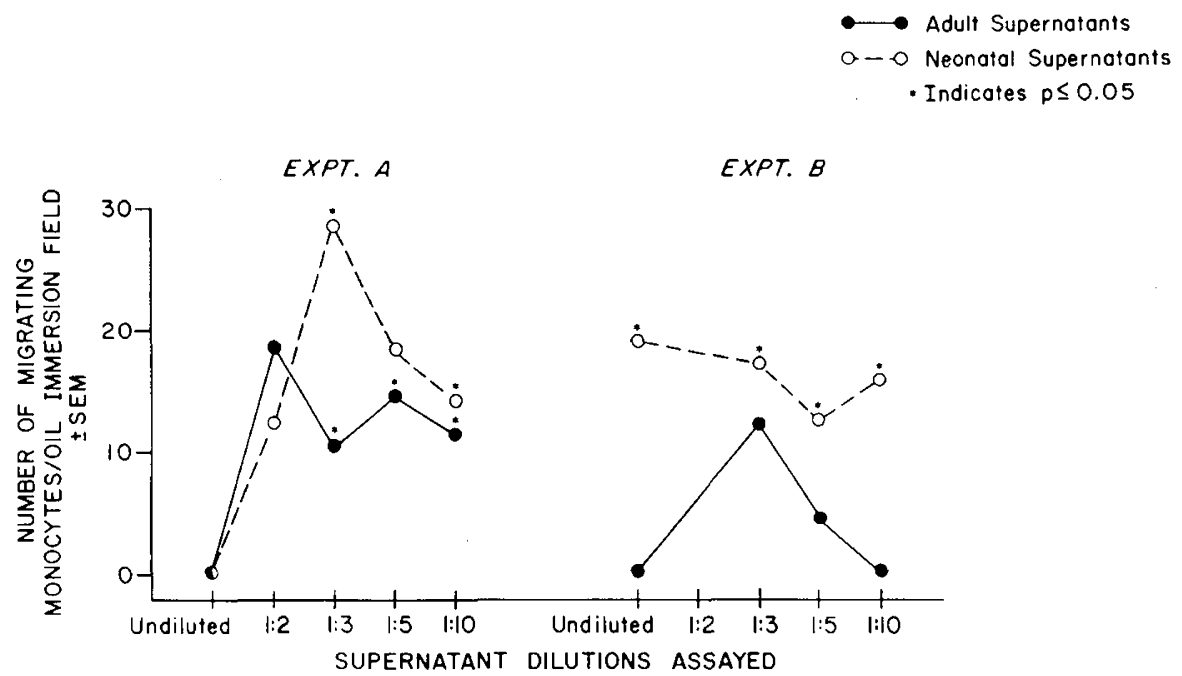

Fig. 1. Dilution assay of neonatal and adult lymphocyte culture supernatants for lymphocyte-derived chemotactic factor activity. The number of monocytes migrating toward stimulated supernatants minus the number migrating toward controls supernatants is shown for each supernatant dilution assayed. Maximal response at each supernatant dilution is shown. Phytohegglutinin $=0.8 \mu \mathrm{g} / \mathrm{ml}$. Asterisk $\left(^{*}\right)$ indicates that the mean number of monocytes migrating toward stimulated supernatants differed significantly from the mean number migrating toward control supernatants when Student's $t$ test $(P \leq 0.05)$ was used.

Other investigators (12) found normal MIF production by cord lymphocytes. Bryson et al. (4) described deficiencies of immune interferon production by both neonatal and cord lymphocytes with normal classical interferon production whereas Handzel et al. (10) found normal immune interferon production by cord lymphocytes. Cord mononuclear cells produced normal LIF (9, 10) but decreased lymphotoxin (7).

Kretschmer et al. (11) examined LDCF production by cord mononuclear cells in a small number of samples. They reported that cord mononuclear cells produced LDCF which was a chemoattractant for adult monocytes but was less chemoattractant for cord monocytes. In view of the reported greater deficiency of neonatal compared to cord mononuclear cells in MIF production (16) and the controversy concerning the functional capability of neonatal cells to produce lymphokines, we chose to extend these earlier studies to include neonatal LDCF production.

Our studies showed neonatal mononuclear cells are functionally as competent as adult mononuclear cells to produce LDCF in response to mitogen challenge. In particular, no quantitative decrease in production of this lymphokine could be demonstrated.
The defect in neonatal skin test hypersensitivity may indeed be more related to deficit cell mobility than deficit lymphokine production. Although cord monocytes chemotax normally, Raghunathan et al. (14) have shown defective neonatal monocyte chemotaxis. Alternatively, antigen-induced production of LDCF by neonatal mononuclear cells may be deficient although mitogeninduced responses appear normal.

\section{REFERENCES AND NOTES}

1. Adelman, N. E., Hammond, M. E., Cohen, S., Dvorak, H. F.: Lymphokines as inflammatory mediators. In S. Cohen, E. Pick and J. Oppenheim, (Eds.): Biology of the Lymphokine, pp. 44-46 (Academic Press, New York, 1979).

2. Altman, L. C., Snyderman, R., Oppenheim, J. J., and Mergenhagen, S. E.: A human mononuclear leukocyte chemotactic factor: characterization, specificity, and kinetics of production of homologous leukocytes. J. Immunol., 110: 301 (1973).

3. Boyum, A.: Separation of leukocytes from blood and bone marrow. Scand. J. Clin. Lab. Invest., 21: 77 (1968)

4. Bryson, Y. J., Winter, H. S., Gard, S. E., Fischer, T. J., and Stiehm, E. R.: Deficiency of immune interferon production by leukocytes of normal newborns. Cell. Immunol., 55: 191 (1980).

5. Cohen, S., Ward, P. A., Yoshida, T., and Burek, C. L.: Biologic activity of extracts 
of delayed hypersensitivity skin reaction sites. Cell. Immunol. 9: 363 (1973).

6. Documenta Geigy. Scientific tables 6th edition. p. 32 (Geigy Pharmaceuticals Summit, 1968).

7. Eife, R. F., Eife, G., August, C. S., Kuhre, W. L., and Staehr-Johanse, K.: Lymphotoxin production and blast cell transformation by cord blood lymphocytes: dissociated lymphocyte function in newborn infants. Cell. Immunol., 14: 435 (1974).

8. Miller, M. E. Host defenses in the human neonate. page 51. (Grune and Stratton, Inc. New York, 1978).

9. Hahn, T., Levin, S., and Handzel, Z. T.: Production of immune and viral interferon by lymphocytes of newborn infants. Isr. J. Med. Sci., 16: 33 (1980).

10. Handzel, Z. T., Levin, S., Dolphin, Z., Schlesinger, M., Hahn, T., Altman, Y., Schechter, B., Shneyour, A., and Trainin, N.: Immune competence of newborn lymphocytes. Pediatrics, 65: 491 (1980).

11. Kretschmer, R. R., Stewardson, P. B., Papierniak, C. K., and Gotoff, S. P.: Chemotactic and bactericidal capacities of human newborn monocytes. J. Immunol., 117: 1303 (1976)

12. Muller, M. R., Lazary, S., and Hitzig, W. H.: Production of migration inhibitory factor and blast cell transformation by cord blood lymphocytes. Int. Arch Allergy Appl. Immunol., 50: 593 (1976).

13. Postlethwaite, A. E. and Snyderman, R.: Characterization of chemotactic activity produced in vivo by a cell-mediated immune reaction in the guinea pig. $\mathrm{J}$. Immunol., 114: 274 (1975).
14. Raghunathan, R., Miller, M. E., Everett, S., and Leake, R. D.: Phagocyte chemotaxis in the perinatal period. J. Clin. Immunol., 2: 242 (1982).

15. Snyderman, R., Meadows, L., and Amos, D. B.: Characterization of human chemotactic lymphokine production induced by mitogens and mixed leukocyte reactions using a new microassay. Cell Immunol., 30: 225 (1977)

16. Stiehm, E. R., Winter, H. S., and Bryson, Y. J.: Cellular (T cell) immunity in the human newborn. Pediatr. Suppl., 64: 814 (1979).

17. Wara, D. W. and Barrett, D. J.: Cell-mediated immunity in the newborn: clinica aspects. Pediatr. Suppl., 64: 822 (1979).

18. Requests for reprints should be addressed to: Dr. Margaret A. Keller, Department of Pediatrics-E6, Harbor-UCLA Medical Center, 10000 West Carson Street, Torrance, CA 90509.

19. This study was supported in part by a Young Investigator Grant from the California Research and Medical Education Fund of the American Lung Association of California, a Public Health Service Young Investigator Research Grant (AI 17124-01) from the National Institute of Allergy and Infectious Diseases, and by NIH-GCRC Grant RR00425. The authors also wish to thank Mr. Edward Smith, Dr. Rukmani Raghunathan and Dr. Michael Miller for their careful review of the Manuscript and Ms. Elaine Praskins for secretarial assistance.

20. Received for publication August 26, 1982

21. Accepted for publication March 1, 1983.

\title{
Failure of High Intensity Auditory Stimuli to Affect Behavioral Arousal in Children during the First Sleep Cycle
}

\author{
K. BUSBY AND R. T. PIVIK ${ }^{(43)}$ \\ Department of Psychiatry and School of Psychology, University of Ottawa, Ottawa, Ontario, Canada
}

\section{Summary}

Behavioral and physiologic indices of arousal to auditory stimuli were examined during the first cycle of sleep in 8-12-year-old hyperactive children and nonhyperactive controls. No behavioral responses or sustained awakenings were obtained for any child during the first cycle of sleep to stimuli at intensities up to $123 \mathrm{~dB}$ sound pressure level $r e 0.0002$ dynes $/ \mathrm{cm}^{2}$ ), i.e., at intensities more than $90 \mathrm{~dB}$ above waking threshold values. Half of the arousal attempts in stage 2 and a quarter of those in stage 4 elicited a partial or momentary physiologic arousal response (i.e., EEG desynchronization and/or change in skin potential response or respiratory activity rates). These arousals were shortlived, with the subjects returning to sleep even with continuing or increased stimulus intensity. Neither the incidence of partial arousals nor the associated threshold intensities differentiated subject groups. Although increased skin potential response activity and decreased respiratory rates were observed during sleep relative to wakefulness, and a predominance of skin potential response activity was noted in stage 4 sleep, no significant differences in frequency (rate/min) of autonomic response measures were obtained when rates before and during auditory stimulation were compared.

\section{Abbreviations}

AATs, auditory arousal thresholds

NREM, non-REM
REMP, rapid eye movement period

SPL, sound pressure level

SPR, skin potential response

$\mathrm{W}$, wakefulness

Normal sleep is punctuated by arousals of varied intensity and duration, which ultimately result in sleep termination and extended wakefulness. This awakening response is one of the most dramatic yet least understood behaviors associated with sleep. Although this response occurs spontaneously i.e., in the absence of disruptive external stimuli, it has been largely through the application of external stimuli in studies determining arousal thresholds from sleep that this process has been examined. These studies have considered varying degrees of arousal, ranging from subawakening variations in physiologic measures to indices of more complete arousal, such as specific behavioral responses indicating stimulus awareness $(2,16,22,27)$, and have established the relevance of several stimulus (modality, intensity, and significance) and state (sleep stage and time of night) variables to the awakening process $(18,24,26,39,40,41)$. In man, these studies have been carried out almost exclusively in adults, and those which have involved children $(20,31,33)$ have not assessed the stimulus parameters required to elicit a behavioral response. The present study provides the first such data and includes the evaluation of physiologic and behavioral correlates of AATs during the first sleep cycle in normal and hyperactive children. The latter 